\section{TNF antibodies get new lease}

Attempts by biotech companies to market antibody-based therapies for septic shock have been repeatedly beaten back by dismal clinical results and a skeptical US Food and Drug Administration (FDA). "It's sepsis 5, biotech 0 ," said one stock analyst, referring to the clinical and regulatory defeats of antibodybased therapies developed by Chiron (Emeryville, CA), Synergen

(Boulder, CO), Centocor (Malvern, PA), Xoma (Berkeley, CA) and Bayer AG (Tarrytown, NY).

But now, one of these companies, Centocor, has resurrected its failed antisepsis product as a potential new treatment for rheumatoid arthritis and Crohn's disease (chronic inflammation of the digestive tract). Centocor has designed a humanized monoclonal antibody (CenTNF) to block the adverse effects of tumor necrosis factor $\alpha$ (TNF- $\alpha$ ), a primary mediator of inflammation. According to Centocor spokesman Bruce Carroll, phase II clinical trials of CenTNF have successfully concluded.

Although Centocor's plans for CenTNF are limited to arthritis and Crohn's disease, recent research suggests that CenTNF may be useful for treating other diseases. For example, louse-borne relapsing fever, an infection caused by the spirochete Borrelia recurrentis, may be a potential target. Patients with relapsing fever have a high mortality rate, so antibiotic therapy is critical for controlling the infection. Unfortunately, antibiotics can sometimes cause a serious inflammatory response known as the Jarisch-Herxheimer (J-H) reaction. This reaction causes sudden fever, rigors, tachycardia and hypotension, and can even be fatal.

Last August, British researchers reported in the New England Joumal of Medicine that antiTNF antibodies could prevent the $\mathrm{J}-\mathrm{H}$ reaction in patients being treated with penicillin. Penicillin ruptures the bacteria and releases components that subsequently activate TNF- $\alpha$ and other cytokines responsible for inflammatory responses. Shutting off TNF with an anti-TNF infusion appears to abort the $\mathrm{J}-\mathrm{H}$ reaction. In the study, 90 percent of relapsing fever patients in a control group had serious reactions. Only 50 percent of those receiving anti-TNF had reactions, and all of those reactions were mild.

Writing in the same issue of the New England Journal of Medicine, sepsis experts Robert Munford and Bruce Beutler (University of
Texas, Dallas) called the study "conceptually clever" and said that "with a minimum of expense these investigators have shown that there is a least one indication for the use of a specific TNF antagonist".

Another possible use for anti-TNF antibody was suggested to David Morrison at the University of Kansas Medical Center after a Lyme disease patient complained reactions following antibiotic therapy. Morrison was an advisor on some of the early studies of antibody therapies for sepsis. Without any clear-cut evidence of efficacy for anti-sepsis antibodies, Morrison began thinking about other less-complicated diseases in which to study antiTNF therapy. Fortunately, the Lyme disease patient kept calling to talk about her symptoms. That led Morrison to think about using the J-H reaction in Lyme patients to study the efficacy of anti-TNF antibodies.

Lyme disease is a nonfatal, tick-borne infection caused by Borrelia burgdorferi, a cousin of the relapsing fever borrelia. Not surprisingly, $\mathrm{J}-\mathrm{H}$ reactions have been documented in Lyme patients receiving antibiotics. In one published case of a patient being treated for neurologic Lyme complications, the resulting J-H reaction was so severe it caused temporary impairment of the patient's eyesight.

\section{Mixed reaction to "research without consent" rule}

The new US government policy allowing research without consent on patients admitted to emergency rooms is likely to have to clear the courtroom first, according to opponents of the measure. The policy, which allows for the enrollment into clinical trials of patients without the patient's consent under certain circumstances, went into effect last month. According to the new US Food and Drug Administration (FDA) rules, "the human subjects (must be) in a life-threatening situation (and) available treatments are unproven or unsatisfactory, and the collection of valid scientific evidence ... is necessary to determine the safety and effectiveness of particular interventions."

"This is a lawsuit waiting to happen," said Robert Veatch of the Kennedy Institute of Bioethics at the Georgetown University in Washington, DC.

That may be true, admits Mary Pendergast, advisor to the director of the FDA, but the government is unlikely to change the policy. In fact, the FDA has granted its first test under the new rules, a trial involving artificial blood in Chicago. According to Pendergast, that trial should begin soon.

Opponents of the new rule say it violates the principles of medical research that were instituted at the Nuremberg trials after World War II, and will challenge it on those grounds. But proponents say it gives much-needed guidance to would-be researchers. "This means that there will be more uniformity and less interpretation of the regulations that exist," said Michelle Biros of the Hennepin County Medical Center.

ERIC WEISSENSTEIN Washington, $D C$ 